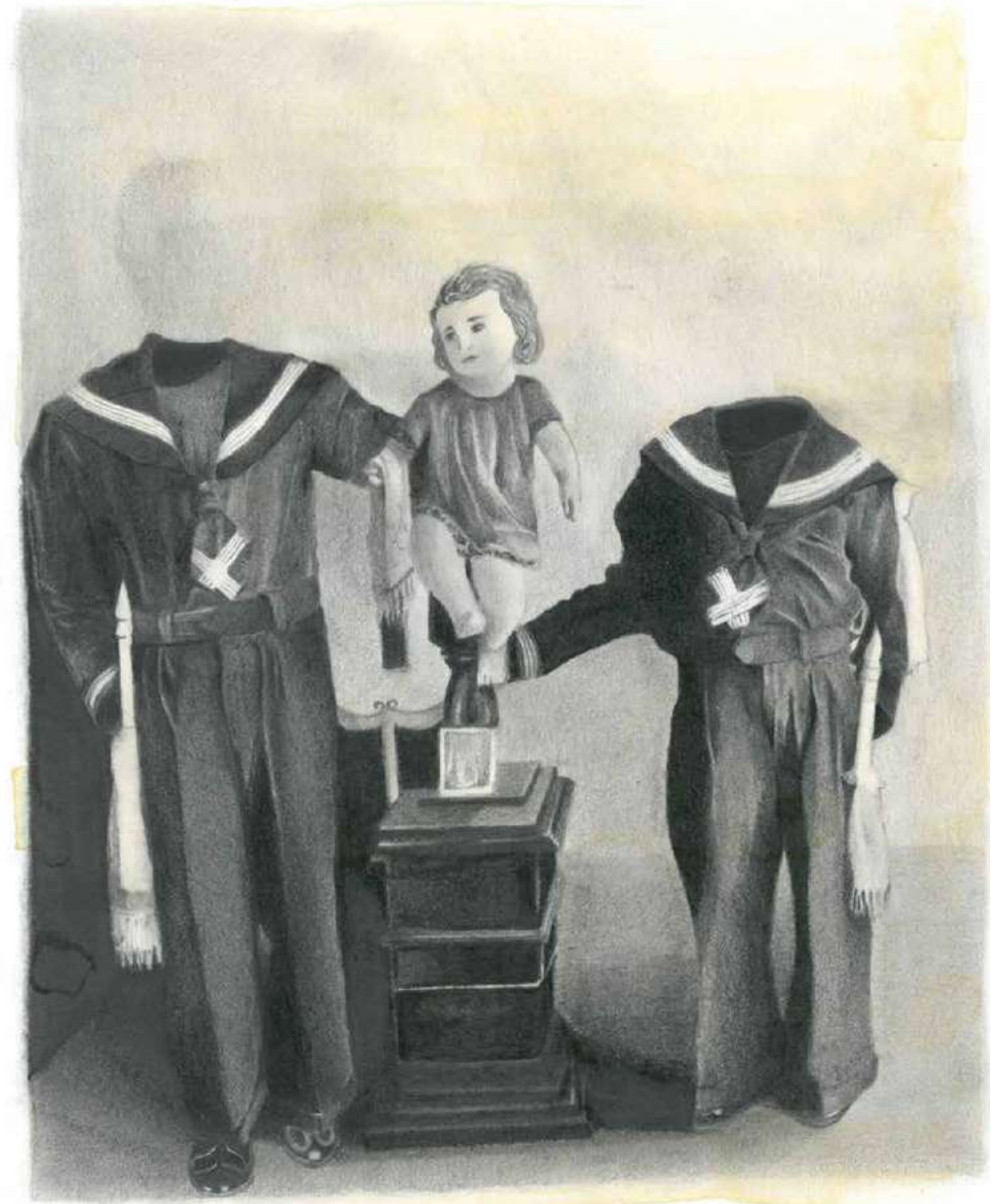

Luis Fernando Salazar Espinosa

Sin título

De la serie Incorpóreo

Lápiz sobre papel

$18 \times 15 \mathrm{~cm}$

2017

Medellín 


\title{
La crisis venezolana y la crisis alimentaria wayuu en Colombia*
}

\author{
Claudia Patricia Puerta Silva (Colombia)*
}

\section{Resumen}

En 2014 se prendieron las alarmas por la muerte de más de 4000 infantes wayuu por hambre en un periodo de seis años. Mediante una revisión de artículos de prensa, reportes de organismos internacionales y nacionales, de literatura académica y con base en un trabajo de campo exploratorio, se identifica la relación entre tres aspectos de la crisis venezolana y el acceso de los wayuu a los alimentos, y se enmarcan en la historia de las dinámicas transfronterizas. La fuerte dependencia de los wayuu de Venezuela y de su aislamiento con respecto a las políticas centralizadas colombianas explica, en parte, que el desabastecimiento de productos de consumo de primera necesidad, la reducción de las remesas y el retorno masivo de población wayuu a sus territorios ancestrales en Colombia contribuyan a la crisis alimentaria que ha afectado desde hace una década a este pueblo indígena.

\section{Palabras clave}

[ 92 ] Relaciones Internacionales; Fronteras; Seguridad Alimentaria; Migración; Población Indígena; Pueblo Wayuu.

Fecha de recepción: junio de 2019 • Fecha de aprobación: noviembre de 2019

\section{Cómo citar este artículo}

Puerta Silva, Claudia Patricia. (2020). La crisis venezolana y la crisis alimentaria wayuu en Colombia. Estudios Políticos (Universidad de Antioquia), 57, pp. 92-114. DOI: 10.17533/udea.espo.n57a05

\footnotetext{
* Este artículo es parte del proyecto de investigación Diseño de un Sistema de Información para el Monitoreo Integral de los Determinantes del Hambre (SINHambre): caso piloto La Guajira indígena, cofinanciado por Colciencias, la Universidad de Antioquia y la Pastoral Social. Una versión inicial fue presentada en las jornadas de estudio Jeux de frontières. De part et d'autres du Venezuela el 5 de junio de 2019 en la École des Hautes Études en Sciences Sociales, París, organizado por Catherine Alès en el marco del programa ECOSnord «Nouvelles formes de participation citoyenne: genre, droits, bureaucratie».

** Antropóloga. Magíster y doctorada en Antropología Social y Etnología. Facultad de Ciencias Sociales y Humanas, Universidad de Antioquia UdeA. Calle 70 No. 52-21, Medellín, Colombia. Correo electrónico: claudia.puerta@udea.edu.co - Orcid: 0000-0002-5105-1073 - Google Scholar: https://scholar.google.es/citations?hl=es\&user=M2qyKsAAAAAJ
} 


\title{
The Venezuelan Crisis and the Food Crisis of the Wayuu People in Colombia
}

\begin{abstract}
In 2014, alarms were raised about the death of more than 4000 Wayuu children from hunger in 6 years. Through a review of press articles, reports from international, national, and academic literature, and based on exploratory fieldwork, the relationship among three aspects of the Venezuelan crisis and the Wayuu's access to food is identified and framed in the history of border dynamics. The strong dependence of the Wayuu on Venezuela and their isolation from centralized Colombian policies partly explain how the shortage of basic consumer products, the reduction of remittances, and the massive return of Wayuu people to their ancestral territories in Colombia contribute to the food crisis that have been affecting these indigenous people in the last decade.
\end{abstract}

\section{Keywords}

International Relations; Boundaries; Food Security; Migration; Indigenous Peoples; Wayuu People. 


\section{Introducción}

En 2014 organizaciones indígenas, entidades gubernamentales y los medios de comunicación alertaron sobre las muertes por hambre de niños y niñas del pueblo wayuu, las cuales continúan sucediendo. Varias razones han sido señaladas: abandono por parte del gobierno colombiano; la corrupción en la atención de salud, nutricional y de servicios básicos; la débil coordinación institucional; el cambio climático; y la crisis económica, social y política en Venezuela.

Mediante la revisión de documentos institucionales, de literatura académica y de prensa, y de un trabajo de campo exploratorio, se identificaron tres aspectos de la crisis venezolana que están relacionados con la disminución de la disponibilidad física de los alimentos y del acceso de los wayuu a estos: a) la falta de disponibilidad de la canasta familiar —alimentos y otros productos básicos-; b) la reducción de las remesas y bienes provenientes de Venezuela; y c) la llegada de población migrante, sean estos venezolanos o los llamados retornados — de nacionalidad colombiana o hijos de colombianos-.

Este trabajo se enmarca en una investigación más amplia que propone

[ 94 ] entender que la crisis venezolana participa en un proceso más amplio y multidimensional que ocasiona hambre entre los wayuu (Puerta et al., 2017). Específicamente, que la crisis del país vecino afecta la disponibilidad física de los alimentos y el acceso económico y físico a estos en Colombia. Afecta así dos de las cuatro dimensiones de la seguridad alimentaria y nutricional según la Organización de las Naciones Unidas para la Agricultura y la Alimentación (FAO). La disponibilidad depende de la producción, el comercio —incluida la importación de alimentos- y la existencia misma del alimento. El acceso es la oportunidad real que tienen las personas de comprar, producir y obtener los alimentos (FAO, 2011; 2016). En cuanto a la seguridad nutricional las dimensiones son la utilización y el consumo (Zúñiga, 2015). En este texto se concentra la atención en las afectaciones sobre la disponibilidad y el acceso.

La crisis venezolana ha sido analizada en términos de seguridad regional o crítica al modelo chavista (Briones y Quishpe, 2019; López Ávila, 2019; López Diaz, 2019; Sutherland, 2019). Se caracteriza por ser política, económica y social. En este texto se asume que la crisis venezolana se manifiesta en el cambio abrupto del patrón migratorio de Venezuela -en 2018 hubo un saldo migratorio negativo, con 3.7 millones de migrantes en 
países receptores liderados por Colombia- y también de la composición de las personas migrantes. Esta se expresa en «la pérdida de bienestar relativo y el grado de amenaza a la subsistencia [...] nueve de cada diez venezolanos manifiestan haber perdido peso en los últimos años» y el aumento de la mortalidad infantil (Gandini, Prieto y Lozano, 2019, pp. 9-10 y 13). La pobreza de $87 \%$ en 2018 , el crecimiento de la pobreza extrema, la expansión del gasto público, el aumento del déficit fiscal y de la deuda pública, una inflación sin precedentes y la contracción del PIB de 35\% entre 2014 y 2018 son otros síntomas de la crisis (Reina, Mesa y Ramírez, 2018; Sutherland, 2019), así como la pérdida de legitimidad del gobierno de Maduro y el aumento de sanciones internacionales (Reina, Mesa y Ramírez, 2018).

Aunque para los wayuu la escasez de alimentos ha sido una constante ligada a periodos de sequía (Ardila, 1990; Gutiérrez de Pineda, 1948; Perrin, 1992; Picon, 1983; Pineda, 1947), desde finales de 2013 se reporta una crisis humanitaria que aún perdura. Para comprender el rol que cumple la crisis venezolana se describe primero la configuración histórica y socioespacial de la frontera y se trata de mostrar la dependencia de los wayuu con la economía y las políticas públicas sociales del vecino país, luego se identifican cuáles son los aspectos de esta crisis que se pueden relacionar con la disponibilidad de alimentos y el acceso de los wayuu a estos.

\section{Configuración sociohistórica de las dinámicas fronterizas}

La frontera compartida Guajira-Zulia ha estado marcada por cuatro fenómenos: «trece etnias, entre ellas la Wayuu, que han reivindicado su condición transfronteriza; conflictos por límites terrestres y marítimos; recursos energéticos que no se aprovechan desde alianzas estratégicas; y dificultades por el contrabando, la corrupción y la inseguridad» (Socorro Ramírez, 2006, citada en Guerra, 2007, pp. 68-69).

Las débiles relaciones que ha tenido el centro gubernamental colombiano con La Guajira explican en buena parte las condiciones de vida wayuu. Al contrario, del otro lado de la frontera fueron muy distintas hasta antes de la crisis de 2013. A pesar de que La Guajira se ha considerado un territorio estratégico por su ubicación geográfica y su situación fronteriza, del lado colombiano ha representado un problema de soberanía. Fundamento de la imaginación geográfica desde el siglo xvı, esta península se ha situado en la frontera de los intereses y políticas públicas, y la ambigüedad de la jurisdicción 
administrativa sobre este territorio ha contribuido a este aislamiento. Al inicio de la conquista, los españoles de Santa Marta no avanzaban en sus expediciones pues temían encontrarse y enfrentarse violentamente con los españoles que venían de la Gobernación de Venezuela (Picon, 1983). Durante la colonia, Riohacha no pudo posicionarse en las dinámicas regionales por estar bajo la jurisdicción de Santa Marta. La ambigüedad de su pertenencia administrativa se constituyó en un conflicto internacional cuando la Gran Colombia se dividió y Colombia y Venezuela se erigieron como países independientes (Puerta, 2013). ${ }^{1}$

Su ubicación de cara al mar Caribe la situó estratégicamente como puente al continente suramericano y específicamente a los Andes. Ha sido un gran puerto natural en donde antes desembarcaron ingleses, franceses, holandeses $y$, actualmente, arubeños, panameños, entre otros. Controlar a los indígenas significaba también controlar sus actividades comerciales con los extranjeros, pero el contrabando siempre puso en peligro las arcas españolas y luego las republicanas, y actualmente afecta las de los Estados modernos colombiano y venezolano (Puerta, 2013).

En los últimos años del gobierno de Álvaro Uribe Vélez y durante el [ 96 ] gobierno de Juan Manuel Santos (2009-2014) se hicieron esfuerzos para ejercer soberanía en la frontera guajira, dada la evidente simpatía de los wayuu con Hugo Chávez. Un funcionario de la Vicepresidencia de Colombia afirmó que la motivación principal de los proyectos de provisión de agua y energía en La Guajira era «ejercer soberanía social en un lugar en donde los indígenas se sentían más reconocidos por el gobierno venezolano». Según él, «el gobierno de Uribe no podía permitirse continuar con la ausencia histórica del estado colombiano en las fronteras y dejar lugar al avance de los simpatizantes del gobierno de Chávez, polo opuesto en la configuración política internacional de América Latina» (Puerta, 2013. Traducción propia).

En la actualidad, La Guajira se describe como una frontera insegura, de «precaria gobernabilidad [y] corrupción en los manejos del erario público [sic]», para la cual no existen políticas específicas, tanto que el tránsito binacional de los wayuu está lejos de formalizarse y muchas de sus actividades comerciales eran calificadas como contrabando. Además, el territorio indígena ha sido despojado o militarizado por la implementación de megaproyectos (Defensoría del Pueblo Colombia, 2014, pp. 76-77).

${ }^{1}$ Los conflictos fronterizos están lejos de estar resueltos, pero no pueden ser objeto de este texto. 


\subsection{Diferencias territoriales: condiciones de vida}

Para 2007, La Guajira contaba con tasas de cobertura de 51,2\% de acueducto, 38,3\% de alcantarillado y $62 \%$ de energía eléctrica, mientras que el estado de Zulia presentaba tasas de $90,7 \%$, 68,1\% y 96,8, respectivamente. Los altos niveles de pobreza estructural —en La Guajira la incidencia de pobreza extrema en 2012 fue cerca de tres veces la pobreza extrema nacional: $27,7 \%$ y 10,4\%, respectivamente (Guerra, 2007, p. 67-68)—, las condiciones de vida de los wayuu del lado colombiano y los índices de violencia comparados con los de sus vecinos, fueron el motivo para que se dieran las dos oleadas de emigración reciente hacia Venezuela —una en la década de 1970, relacionada con la bonanza marimbera y un auge de la economía venezolana por el petróleo, y la de la década de 1990 e inicios de 2000, por el conflicto armado en la zona-. ${ }^{2}$

Junto con los alimentos, el combustible era adquirido del otro lado de la frontera, así como el agua. Una líder de Portete, indignada afirmó que del lado venezolano había un tubo que Ilevaba agua hasta la Alta Guajira mientras que en Colombia solo pasaban tubos de gas y que los wayuu toman agua salada (Puerta, 2013).

Otro aspecto en el cual las diferencias siempre fueron motivo de comparación es en la atención y servicios de salud, especialmente después de 1993, cuando el sistema de salud colombiano pasó a un esquema de subsidio a la demanda con barreras burocráticas al acceso (Abadía y Oviedo, 2010; Dover y Puerta, 2008). Del lado venezolano se continuó con un esquema subsidiado a la oferta por parte del Gobierno central y se fortaleció la atención intercultural. Antes de la crisis los wayuu transitaban de un lado de la frontera al otro, de acuerdo a las necesidades médicas (Dover y Puerta, 2008; Puerta y Dover, 2007). La población wayuu demandaba la presencia médica y de medicamentos en puestos de salud distribuidos por el territorio, o de brigadas médicas frecuentes, especialmente para evitar el traslado de los habitantes

\footnotetext{
${ }^{2}$ La primera gran oleada de emigración hacia Venezuela se dio desde la segunda mitad del siglo XIX hasta principios del siglo $\mathrm{xx}$, cuando se comenzaron a explotar los yacimientos de petróleo de Maracaibo y las haciendas de la región se quedaron sin trabajadores. En ese momento la migración voluntaria se dio al mismo tiempo que una trata de esclavos wayuu (Pérez, 2004; Perrin, 2003; Puerta, 2013). A mediados del siglo xx hubo una emigración significativa relacionada con las largas sequías que soportaron los wayuu del lado colombiano, los cuales se dirigieron a Maracaibo (Chaves, 1951; Pineda, 1947).
} 
de la Alta Guajira a los centros de salud de Maracaibo, Paraguaipoa, Uribia y Nazareth (Guerra, 2007, p. 212).

Finalmente, la debilidad institucional y la muy reciente participación de los wayuu en política del lado colombiano también representan una diferencia significativa en esta frontera. La oferta institucional social venezolana fue constante hasta la crisis y los wayuu del lado venezolano formaban parte de algunos sectores de la clase política e intelectual de Zulia, lo cual fue evidente en su alta participación en los últimos gobiernos venezolanos, especialmente de Chávez (Alarcón, 2006).

\subsection{Binacionalidad}

En esta frontera habitan trece grupos indígenas, del cual el wayuu es el más numeroso. Ambos gobiernos aceptaron su binacionalidad y, en consecuencia, la doble documentación y el libre tránsito de los miembros de la etnia —desde 1991 en Colombia y desde 1999 en Venezuela-. En principio, serían libres de ocupar y transitar por su territorio sin reconocer las fronteras político-administrativas trazadas por los dos países (Guerra, 2007, p. 70). De hecho, «los wayuu habían transitado constantemente entre los dos

[ 98 ] territorios en búsqueda de mejores condiciones de vida, que hasta hace poco estaban del lado venezolano» (p. 69).

En reconocimiento de la ancestralidad del pueblo wayuu y de su dependencia a la economía venezolana, los gobiernos llegaron a algunos acuerdos que incluían la participación de cooperativas indígenas en el comercio legal de combustible y de productos de la canasta familiar. En 2007 estaba vigente en Colombia la normativa sobre comercio de alimentos, llamada Ley de Fronteras (Ley 191 de 1995), que permitía la introducción de bienes provenientes de cada país colindante exclusivamente para consumo dentro de las Unidades Especiales de Desarrollo Fronterizo. En La Guajira, el ingreso de productos de la canasta familiar y de combustible desde Venezuela se daba a través de cooperativas indígenas, esquema de trabajo impuesto por el marco legal colombiano. Se conformaron por lo menos cuatro cooperativas: Wayuucoop, Shiliwalacoop, Tawalacoop y Estrewacoop. La Resolución 01922 del 23 de febrero de 2007 de la Dirección Nacional de Impuestos y Aduanas Nacionales (DIAN) establecía una lista de bienes y los cupos máximos semestrales que se podían ingresar por Paraguachón «hacia 
los depósitos privados habilitados por la DIAN pertenecientes a la comunidad indígena Wayuu» (Guerra, 2007, p. 153).

Tabla 1. Cupo semestral de productos para el comercio wayuu, 2007.

\begin{tabular}{|c|c|c|c|c|c|}
\hline Alimentos básicos & Cantidad & Unidad & $\begin{array}{c}\text { Productos básicos } \\
\text { de aseo }\end{array}$ & Cantidad & Unidad \\
\hline $\begin{array}{l}\text { Preparaciones para la } \\
\text { alimentación infantil a } \\
\text { base de leche }\end{array}$ & 200 & Toneladas & Papel Higiénico & 3000000 & $\begin{array}{l}\text { Rollos } \\
\text { normales }\end{array}$ \\
\hline Arroz & 5484 & Toneladas & Jabón de tocador & 300 & Toneladas \\
\hline Harina de trigo & 1750 & Toneladas & $\begin{array}{l}\text { Jabón en barra } \\
\text { para lavar }\end{array}$ & 400 & Toneladas \\
\hline Harina de maíz & 1500 & Toneladas & Jabón en polvo & 400 & Toneladas \\
\hline Azúcar & 3838 & Toneladas & $\begin{array}{l}\text { Crema dental o su } \\
\text { equivalente }\end{array}$ & 80000 & Galones \\
\hline Aceite & 1093 & Toneladas & Champú & 80000 & Galones \\
\hline $\begin{array}{l}\text { Preparaciones a base de } \\
\text { cereales }\end{array}$ & 500 & Toneladas & & & \\
\hline Salsas & 150 & Toneladas & & & \\
\hline Sazonadores & 2 & Toneladas & & & \\
\hline $\begin{array}{l}\text { Pollo sin trocear, } \\
\text { refrigerados o } \\
\text { congelados }\end{array}$ & 500 & Toneladas & & & \\
\hline Huevos & 20000 & Docenas & & & \\
\hline
\end{tabular}

Fuente: elaborado de partir de DIAN (Resolución 01922 de 2007).

La mayor parte de los productos de la canasta familiar y los bienes para consumo animal estaban exentos de IVA. Esta medida había favorecido el acceso a los alimentos para los wayuu, «especialmente porque los productos de la canasta familiar en Venezuela son subsidiados por el gobierno» (Guerra, 2007, p. 154).

Pese a la intención de estas medidas que permitían la participación de los wayuu en dinámicas comerciales de la frontera, estas se cuestionaron por la infiltración del conflicto armado colombiano, el narcotráfico y el contrabando. 


\subsection{Contrabando}

Dos tipos de contrabando coexisten en La Guajira: el contrabando «democratizado» o realizado por un gran número de personas que traficaban, antes de la crisis, pequeñas cantidades de divisas, gasolina, productos de la canasta familiar, textiles y otros; y el controlado por grupos ilegales y que es de mayor escala (González-Plazas, 2008). El primer tipo es de origen colonial, en él participan amplios sectores de la sociedad guajira y tiene como objeto «pequeñas cantidades de productos de consumo masivo y de alimentos». Indígenas, otros guajiros y pequeños comerciantes contrabandeaban por sus propios medios productos de la canasta familiar (p. 95).

Este texto no se puede detener en las razones de la preponderancia en la economía regional de actividades que pueden considerarse informales e ilegales; sin embargo, es de toda la importancia para el análisis de la crisis alimentaria:

La generalización de actividades ilícitas como modo de subsistencia, en esta región confluyen una serie de aspectos que así lo permiten, entre ellos, el carácter semidesértico y las grandes extensiones de tierras con baja densidad poblacional, la existencia de puertos naturales, la mayoría controlados por los indígenas, la falta de oportunidades económicas, la lejanía del centro del país y la tradición de comerciantes de la población [...] (Guerra, 2007, p. 71).

Estas actividades de contrabando y de comercios informales han tenido hitos en la «bonanza marimbera» y, más recientemente, en el tráfico de armas y el narcotráfico (Defensoría del Pueblo Colombia, 2014, pp. 96-97). Maicao se desarrolló como puerto libre en la década de 1970, pero en la década de 1990, por los controles de la DIAN y la apertura económica, perdió su liderazgo y entró en crisis. Se siguió realizando el contrabando en buses y carros de pasajeros, pero este «contrabando» o comercio informal fue uno de los argumentos utilizados para la decisión que tomó Nicolás Maduro para cerrar la frontera. Era bien sabido que era significativa «la diferencia de precios de la gasolina o de productos de la canasta familiar por los subsidios del lado venezolano» (González-Plazas, 2008, p. 96). Tanto este comercio informal como el contrabando de telas, cigarrillos y licores se acabaron en la región, y los grandes y pequeños contrabandos de droga, gasolina, armas y carros robados fueron acaparados por bandas criminales organizadas. El contrabando «malo» remplazó al contrabando «bueno» (p. 103). 
Este segundo tipo de contrabando es de gran escala y controlado por grupos ilegales organizados. El departamento de La Guajira es un «corredor estratégico de movilidad y aprovisionamiento de armas, gasolina de contrabando e insumos para la elaboración de narcóticos...» (Defensoría del Pueblo Colombia, 2014, p. 73). Diferentes organizaciones determinaron la presencia de grupos al margen de la ley en las zonas fronterizas. Los Rastrojos grupo neoparamilitar o banda criminal (bacrim) — controlan la zona de la Alta Guajira en Colombia y de Zulia en Venezuela. Hasta la firma de los acuerdos de paz con las Fuerzas Armadas Revolucionarias de Colombia-Ejército del Pueblo (FARC-EP), esta guerrilla controlaba la zona de la serranía del Perijá (SJR, 2015, p. 11). Las dinámicas del narcotráfico atrajeron a Los Urabeños otro grupo neoparamilitar o bacrim - , quienes sostienen un conflicto con Los Rastrojos y que del lado venezolano se encuentran en Maracaibo, Ureña y San Cristóbal. En la zona fronteriza también se han detectado enfrentamientos entre las Fuerzas Bolivarianas de Liberación — grupo venezolano al margen de la ley-y el Ejército de Liberación Nacional (ELN) de Colombia (p. 11). ${ }^{3}$

En este contexto, las cooperativas indígenas de lado y lado de la frontera que se constituían como alternativas económicas no duraron mucho en ser cooptadas por los paramilitares y bandas emergentes (González-Plazas, 2008, p. 98); además, este tipo de actividades hacen parte del proceso de despojo, pues acaparan territorios para sus corredores estratégicos:

Hay pueblos de La Guajira que huelen a gasolina. Porque en muchos de ellos, sobre todo en los del norte, hay más gasolina ilegal que agua. Es decir, circula más líquido para poner a funcionar un carro del que se necesita para mantener con vida a los niños [...].

El negocio de la gasolina, que comenzó hace más de diez años como una ayuda del presidente Hugo Chávez a los wayúu [sic], se ha convertido en una gran multinacional del crimen. No solo puede mover entre 2,5 y 5 billones de pesos de utilidades al año, sino que ha devastado lo poco que quedaba de instituciones. Gracias a ella algunos clanes y bandas armadas se tomaron esa inhóspita región (Semana, 2014, julio 19).

La caracterización de las fronteras como zonas peligrosas e incontrolables entra a mediar las relaciones centro-periferia mediante las que el Estado

\footnotetext{
${ }^{3}$ No podrá ser objeto de este texto, pero hay noticias de la incursión tanto de las FARC-EP como del ELN hasta los territorios indígenas en Venezuela en la cuenca del Orinoco, ejerciendo el control de la minería y el narcotráfico (Alès, 2018).
} 
colombiano hace presencia en la región. Durante años el contrabando ha sido uno de los términos más usados para referirse a La Guajira y, al mismo tiempo, es una de las actividades que más ha estimulado su economía, pero también la reciente presencia de grupos armados ilegales complejiza la situación fronteriza.

Y es que el contrabando «malo» no es tan fácilmente distinguible del «bueno» o del comercio informal, como algunos también lo caracterizan. Varios estudios y la prensa indican «la proclividad de las autoridades venezolanas y colombianas a ser sobornadas», fenómeno que contribuye al desarrollo de este contrabando (Consejero de fronteras del Gobierno Venezolano, 2007, citado en González-Plazas, 2008, p. 99). Se señala además que es «infortunadamente, a partir de este contrabando [...] que buena parte de la población de La Guajira y de departamentos aledaños, incluido Norte de Santander, se alimenta» (González-Plazas, 2008, p. 99).

\section{Factores de la crisis venezolana que inciden en el acceso de los wayuu a los alimentos}

Como se ha dicho, el inicio de la crisis venezolana se sitúa a principios

[102 ] de 2013 y se han identificado tres aspectos de esta que inciden en la escasez de alimentos y otros bienes esenciales para la subsistencia de los wayuu: a) la falta de suministros de alimentos y otros productos básicos; b) la reducción de remesas de Venezuela a Colombia; y c) el ingreso de población migrante, ya sea venezolana o colombiana que regresa a su lugar de origen.

\subsection{Escasez de alimentos y de otros productos básicos}

En cuanto a los alimentos y otros bienes, «el Gobierno venezolano se ha concentrado en la lucha contra el contrabando al considerar que este es una de las causas principales de la escasez y desabastecimiento que vive el país» (SJR, 2015, p. 6). Las mafias del bachaqueo — mercado negro en Venezuelapreocupan al gobierno venezolano. Es una práctica de contrabando y desvío de fármacos, alimentos y otros rubros de la cesta básica; sin embargo, era una práctica consolidada entre los wayuu: «La detención en Venezuela de 110 mujeres de la etnia por el delito de contrabando es un «atentado» a sus derechos, pero sobre todo a sus usos y costumbres [...] estas mujeres fueron detenidas por llevar arroz, aceite, harina o cualquier otro alimento que acostumbran a comprar en Venezuela para traer a sus familiares en Colombia» (El Heraldo, 2014, septiembre 27). 
El conjunto de medidas tuvo un impacto en la ya precaria situación de La Guajira «debido a la dependencia económica histórica que se presenta en este lugar de la frontera» (Bonet-Morón y Hahn-de-Castro, 2017). El desabastecimiento de gasolina también aumentó el costo de productos de la canasta familiar y del transporte (Guerra, 2007, p. 181).

Ya la sequía prolongada y la debilidad institucional del lado colombiano estaban «afectando a las comunidades Wayúu [sic] de la Alta Guajira de forma crónica, debilitando las redes sociales de apoyo de las familias y sus medios de subsistencia (agricultura y pastoreo) e incrementando la dependencia de estas comunidades/familias de los ingresos económicos y la oferta institucional ofrecida por el gobierno de Venezuela» (OCHA, 2014, p. 2). Por ello fue tan contundente el efecto del cierre de la frontera. La Defensoría Regional de La Guajira llamó la atención de la Unidad de Gestión del Riesgo para llevar alimentos a 24000 familias en los meses de febrero a mayo de 2014 (Defensoría del Pueblo Colombia, 2014, p. 138). Se estableció un Comité Binacional de Control de Comando y Control Fronterizo conformado por representantes de ambos lados de la frontera, incluyendo medidas como: el despliegue de las fuerzas armada, la restricción del tránsito de carga y cierre de la frontera en las noches, y «la prohibición de exportación de todos los productos de la canasta básica venezolana por vía de decreto del poder ejecutivo venezolano» (SJR, 2015).

La histórica dependencia económica (Viloria de la Hoz, 2014) explica por qué la actual escasez de alimentos y productos básicos que se observa en Venezuela afecta gravemente a las familias wayuu (Bonet-Morón y Hahnde-Castro, 2017, p. 21). Especialmente en la Alta Guajira, región aislada de los centros urbanos y de los mercados, los alimentos provenían de Venezuela a precios accesibles. Se complementaba la compra directa con redes de apoyo, el tránsito de parientes y el envío de remesas y de encargos. El desabastecimiento por las restricciones de Venezuela (Guerrero, 2014, abril 7) y el cierre de la frontera afectó las estrategias de afrontamiento que los wayuu habían activado históricamente en tiempos de escasez. La Guajira nunca pudo consolidar una suficiencia agrícola en el siglo xIx (Viloria de la Hoz, 2014), lo que se agravó en el siglo xx con el extractivismo mineroenergético que aún contribuye al despojo, restricción y afectación por contaminación, tanto del territorio como de los recursos para la subsistencia autóctona (Puerta, 2018). 
De hecho, antes de la crisis, las cancillerías de Colombia y Venezuela durante los gobiernos de Álvaro Uribe y Hugo Chávez acordaron medidas para atenuar el hambre de los guajiros.

Chávez autorizó que camiones colombianos compraran productos subsidiados de los mercados de su país para vender al pueblo guajiro. El acuerdo tenía dos ventajas: los alimentos valían una quinta parte y era más fácil proveer ciertas zonas de La Guajira que son muy distantes de cualquier ciudad intermedia de Colombia. Pero también acarreaba un problema, pues el negocio era tan bueno, que el número de camiones creció y se podía encontrar queso salado de Zulia en Córdoba. En abril de este año, debido a la crisis alimentaria en Venezuela, se puso fin a ese acuerdo.

El personero de Uribia, Enrique Barros, denunció hace poco que los indígenas que años atrás consumían aceite y arroz que entraba por Puerto Estrella y Siapana, ahora solo están comiendo chivo. Y eso cuando hay (Guarnizo, 2014, julio 19).

Cuando se cerró la frontera tanto el negocio lucrativo que denunciaba el gobierno de Maduro como el acceso a alimentos a bajos costos para los wayuu se redujo notablemente: «Las medidas cambiarias y la regulación de la participación del sector privado en la producción y distribución de algunos productos básicos han desencadenado una escasez de productos básicos, presiones inflacionarias y problemas de suministro en un aparato productivo altamente dependiente de las importaciones» (Bonet-Morón y Hahn-deCastro, 2017, p. 20).

Las principales consecuencias de la sequía habían sido la desaparición de todo tipo de agricultura a partir de la mitad de 2013, la reducción del número de animales por familia, una progresiva descapitalización y el incremento de la dependencia del comercio y de los mercados locales venezolanos (Raffalli y Padrilla, 2014, p. 23). Dado que, en general, los wayuu cuentan con la ganadería y las remesas de Venezuela como sus principales fuentes de ingresos, la crisis venezolana actuó como un hold de la crisis alimentaria de los wayuu (Howe, 2018).

El desabastecimiento de alimentos y de productos de higiene y aseo (OCHA, 2014, p. 5) no afectó solamente el comercio, sino también los envíos que los familiares hacían hacia Colombia. Como se anotó antes, las restricciones afectaron igualmente los viajes que desde Colombia hacían 
las mujeres para abastecerse. Cuando el cierre de la frontera, la Oficina de Naciones Unidas para la Coordinación de Asuntos Humanitarios (OCHA) lanzó alertas para atender a las poblaciones wayuu: «Persiste necesidad de asegurar acceso a alimentos a las poblaciones que han visto afectados sus medios de vida y acceso a alimentos por el cierre de frontera, en particular comunidades rurales de Uribía [sic] y Maicao» (OCHA, 2015, p. 7). En 2015, cuando se ordenó cerrar la frontera y se comenzaron a deportar colombianos masivamente, el comercio se afectó. Si bien en el segundo semestre de 2016 se reabrió la frontera, la crisis económica de Venezuela se materializó en escasez de alimentos, hiperinflación y la devaluación acelerada del Bolívar y la emigración masiva (Reina, Mesa y Ramírez, 2018).

No solo los alimentos dejaron de movilizarse a través de la frontera: «Se mantienen restricciones para el acceso a agua en poblaciones rurales que adquieren agua en Venezuela y se ven afectadas por el cierre de frontera, situación agravada por la sequía prolongada en el departamento que además tiene consecuencias sobre la salud, seguridad alimentaria y nutrición» (OCHA, 2015, p. 10). Y el desabastecimiento de combustible «también afecta el suministro de energía a los centros poblados e igualmente de los centros de salud y del hospital, así como el consumo de agua potable en tanto las motobombas y plantas desalinizadoras requieren combustible para su funcionamiento» (OCHA, 2014, p. 8). Asimismo, los cierres de la frontera afectaron la cría de animales, pesca y artesanía: «Preocupa la falta de fuentes de trabajo y pérdida de animales de pastoreo en comunidades rurales que basaban su economía en la cría de animales, pesca y artesanía, actividades que se han visto limitadas por los cierres de frontera, temas climáticos, falta de herramientas e insumos» (OCHA, 2015, p. 11).

\subsection{Casi completa desaparición de las remesas}

En lo que respecta a las remesas oficiales o legales —institucionalizadas«los giros de Venezuela a Colombia cayeron sustancialmente entre 2014 y 2015. Al inicio de 2014 Venezuela restringió los giros internacionales: se restringió la cantidad, se aumentó el valor cambiario del dólar y se impusieron nuevas condiciones» (Noticias RCN, 2014, enero 27). La caída de las remesas se sintió con más fuerza, pues entre 2005 y 2013 habían aumentado excepcionalmente por el incremento de emigración colombiana y «la posibilidad de arbitraje derivada del diferencial entre la tasa de cambio oficial y la tasa del mercado negro que existía en las calles» (Reina, Mesa y 
Ramírez, 2018). Aunque no solo las remesas en dinero desaparecieron, los familiares del lado venezolano dejaron también de enviar remesas en especie, «afectando el nivel de ingreso de las familias» (OCHA, 2014, p. 9), pues habían «sido una fuente importante de recursos a lo largo de la historia para los hogares colombianos» (Reina, Mesa y Ramírez, 2018). Con las remesas, el comercio bilateral cayó a sus más bajos niveles.

Ya se mencionó la escasez de alimentos venezolanos, tanto al interior de ese país como del lado colombiano. La disponibilidad de alimentos, según la $\mathrm{FAO}$, es función, entre otras, del comercio. Este se ha visto afectado entre Colombia y Venezuela desde finales del siglo xx por las dificultades en las relaciones diplomáticas. Luego de 2008 se desplomó y aunque se recuperó un poco volvió a caer en 2013, debido al cierre fronterizo y al estado de excepción en 2015 en Venezuela (Reina, Mesa y Ramírez, 2018). Sumado a la prohibición a la exportación de alimentos, a los wayuu solo les quedó la opción de comprar alimentos más costosos en Colombia. En Uribia y Maicao las tiendas estaban bien surtidas con productos colombianos, pero los venezolanos eran pocos. El acceso al alimento se vio afectado porque los recursos de las personas no fueron suficientes para pagar el costo de los productos colombianos (OCHA, 2014, p. 2). En conclusión, las diferentes fuentes de ingreso de la población de la zona se afectaron por la coyuntura de restricción del comercio con Venezuela (p. 8).

\subsection{Migración masiva a La Guajira y retorno a los territorios wayuu}

El tercer elemento, la migración hacia Colombia, actúa también en la crisis alimentaria. A principios de 2013, la percepción de que había mejores condiciones de ese lado de la frontera da un giro dramático. Desde 2014 se registraron las oleadas más numerosas de inmigración a Colombia, ya sea retorno o deportación de la población colombiana a sus sitios de origen, o de venezolanos, la cual no hace sino aumentar (Semana, 2018, octubre 2).

Existe una gran cantidad de familias colombianas que regresan desde Venezuela por la aguda situación política y económica, algunas inclusive han sido deportadas, estas circunstancias afectan al municipio de Maicao principalmente debido a que recibe aproximadamente 5 integrantes por núcleo familiar incluidos niños y niñas que en muchas ocasiones coinciden con ser personas víctimas de la violencia (Defensoría del Pueblo Colombia, 2014, p. 19). 
El desplazamiento forzado por el conflicto armado del pueblo wayuu hacia Venezuela a partir de la década de 1990 ha sido invisibilizado: por un lado, por ser un pueblo binacional que normalmente migra por épocas hacia Venezuela, en donde unos tienen familiares, otros sus viviendas; en el vecino país se atienden en salud y beneficios que reciben del Gobierno venezolano; por el otro, las autoridades locales departamentales y municipales relacionaban los casos de desplazamientos de indígenas wayuu con estas migraciones normales hacia Venezuela (Defensoría del Pueblo Colombia, 2014, p. 74). Hay que recordar que las oleadas en la década de 1970 por razones económicas y de principio del siglo $\mathrm{xx}$ al inicio de la explotación del petróleo también incluyeron migraciones voluntarias y forzadas.

En definitiva, el regreso de colombianos es otro factor que ha contribuido a la agudización de la crisis humanitaria en este departamento. También llegan a Colombia venezolanos con la expectativa de generar ingresos en pesos y que el cambio al Bolívar aumente el monto de lo producido, así como en épocas anteriores en que los colombianos cruzaban la frontera hacia Venezuela con la expectativa de encontrar mejor calidad de vida (Defensoría del Pueblo Colombia, 2014, p. 74).

Como se mencionó ya, la migración hacia Venezuela ha sido común entre los wayuu y se pueden identificar por lo menos tres grandes oleadas: alrededor de 1910-1920, cuando se inició la explotación petrolífera y se da una trata de esclavos indígenas para cubrir las necesidades de las haciendas, así como una migración económica voluntaria. En la década de 1970 el país vecino era boyante y otra oleada de migración puede ser identificada con la bonanza marimbera colombiana. Finalmente, en las décadas de 1990 y 2000 la migración es por la violencia — se recuerdan las masacres en el sur de La Guajira y en Portete-.

Los wayuu prefieren hablar de retorno que de migración, pues muchos de ellos reivindican su ancestralidad dada la ubicación de su cementerio matrilineal en territorio del lado colombiano.

Los indígenas retornados - que en su mayoría vivían en el Estado Zulia, en Venezuela-, se instalaron en los municipios colombianos de Uribia, Maicao, Manaure y Riohacha. Viven en asentamientos que crearon y en las rancherías de sus hermanos establecidas a lo largo y ancho del desierto. 
Según datos del Registro Administrativo de Migrantes Venezolanos, realizado por la Unidad Nacional de Gestión de Riesgo entre abril y junio de [2018], a La Guajira Ilegaron 74874 migrantes. En todo el país, quienes se reconocen como indígenas provenientes de Venezuela suman 20579 (Liga Contra el Silencio, 2018, noviembre 30).

La integración de los «retornados» no ha sido fácil. Sufren de discriminación por parte de vecinos y parentelas, y del doble desarraigo de sus territorios ancestrales y de sus territorios de vida. Se les aísla de las actividades cotidianas y se les recrimina por no haber contribuido a los asuntos familiares durante sus años de ausencia. Algunos «retornados» son considerados achon, haciendo referencia a que retornan y reivindican derechos asociados al territorio paterno, lo cual es una excepción a la matrilocalidad wayuu. Y el retorno del wayuu a sus territorios de origen debilita las redes de apoyo con las que contaban las parentelas, pues ya estas personas no envían remesas ni mercados ni mercancías desde Venezuela, con lo que se afectan las economías familiares.

Algunas autoridades señalan que el aumento de la población exige mayores recursos. El suministro de agua ya no es suficiente: «cada ocho días el Cabo de la Vela era abastecido con dos carrotanques que ya no son suficientes para la alta demanda. En lo que va del año, 15 familias migrantes (60 personas) se han asentado solo en este sector» (Liga Contra el Silencio, 2018, noviembre 30).

La ocupación de sectores aledaños a Uribia y a Maicao preocupa por las pésimas condiciones de estos nuevos asentamientos. Los programas de ayuda humanitaria no dan abasto. Sin embargo, algunas autoridades tradicionales han recibido a parientes y aliados en sus territorios familiares:

David Rodríguez Viloria, autoridad tradicional del e'iruku (clan) Epiayú, quien en el último año ha recibido en su casa en Uribia entre 10 y 12 familias venezolanas al mes, recuerda mejores tiempos. «Todas las semanas nos mandaban encomienda -alimentos y mercancía- en unos buses que le decían "termotabla" o chirrinchera. Ahora debemos retribuirles», añade (Liga Contra el Silencio, 2018, p. 8).

Esta autoridad se refiere a lo que se mencionó anteriormente: el continuo intercambio y flujo de bienes, remesas y personas que de un lado y otro de la frontera complementaban las economías domésticas de las parentelas wayuu. 
Pero estos flujos son, hoy en día, de alto riesgo. Con los cierres y restricciones fronterizas, más de doscientas trochas se han abierto en territorio indígena en donde tanto personas wayuu como no indígenas se han instalado para cobrar peajes a quienes intentan pasar. Violencia y atropellos sufren las personas que han habitado esta zona y quienes intentan pasar por allí.

\section{Conclusiones}

El aislamiento socioespacial histórico con el centro colombiano es proporcional a la estrecha articulación socioespacial histórica de La Guajira con Venezuela. Esta paradoja se evidencia aún más cuando este último se ve inmerso en una profunda crisis que revela la dependencia de los wayuu con respecto a los bienes de consumo básico, al agua, al combustible, a las remesas, al intercambio de bienes y a la migración laboral disponibles en Venezuela, así como a las carreteras del vecino país para movilizarse desde la Alta Guajira hasta centros urbanos como Uribia y Maicao y, antes de la crisis, a Maracaibo.

A partir de esta revisión, las dificultades actuales para la disponibilidad y el acceso a los alimentos están relacionadas con varios elementos: a) la ruptura de los acuerdos existentes para que los wayuu pudieran comerciar con productos de la canasta familiar a través de cooperativas y transportar pequeñas cantidades de alimentos gracias a su condición binacional; b) el cierre de las fronteras: prohibición y castigo del transporte de productos de la canasta familiar en pequeñas cantidades de personas que viajan con frecuencia entre los dos países y afectación de la atención en salud, pues las personas no pueden transitar por las carreteras de Venezuela para acceder más rápidamente a cascos urbanos en épocas de lluvia, por ejemplo; c) el aumento de los costos de los productos de la canasta familiar asociados a las dinámicas del combustible — cuando hay desabastecimiento se aumentan los costos de los alimentos o escasean los mismos porque no se pueden transportar - y al desabastecimiento en Venezuela; d) la reducción de las remesas y especies provenientes de Venezuela; e) el retorno de los wayuu a los territorios de origen, rompiendo y debilitando las redes solidarias parentales transfronterizas, aumentando la presión demográfica en condiciones ya precarias y propiciando conflictos territoriales entre parentelas y al interior de ellas; y f) las dinámicas del conflicto armado, la presencia de grupos paramilitares, guerrilleros, bacrim, narcotraficantes y delincuentes comunes. 
En suma, la crisis venezolana es un hold en la crisis alimentaria wayuu que les afecta desde 2012, en la que inciden otros factores de larga duración y eventos de menor duración que actúan como estresores de una situación ya precaria para los wayuu acceder a sus alimentos. Este fenómeno multidimensional del hambre en La Guajira exige una comprensión procesual —en el tiempo- y socioespacial más allá de las fronteras políticoadministrativas. Con este artículo se logra mostrar la naturaleza regional de las dinámicas de la sociedad wayuu y las implicaciones de las crisis globales en lo más íntimo de las familias indígenas.

\section{Referencias bibliográficas}

1. Abadía Barrero, César Ernesto y Oviedo Manrique, Diana Goretty. (2010). Itinerarios burocráticos de la salud en Colombia: la burocracia neoliberal, su estado y la ciudadanía en salud. Revista Gerencia y Políticas de Salud, 9 (18), pp. 86-102.

2. Alarcón Puentes, Johnny. (2006). La sociedad wayuu, entre la quimera y la realidad. Gazeta de Antropología, 22. Recuperado de http://hdl.handle. net/10481/7098

3. Alès, Catherine. (2018). From Proclamation to Denial. Regions and Cohesion, 8 (2), pp. 49-81. DOI: 10.3167/reco.2018.080204

Ardila Calderón, Gerardo. (1990). La Guajira. Bogotá, D. C.: Universidad Nacional de Colombia.

4. Bonet-Morón, Jaime y Hahn-de-Castro, Lucas Wilfred. (2017). La mortalidad infantil en La Guajira: Una caracterización estructural. Documentos de Trabajo sobre Economía Regional, 255. DOI: 10.32468/dtseru. 255

5. Briones Aguirre, Renato Josué y Quishpe, Jeverson Santiago. (2019). Análisis ético de la crisis humanitaria en Venezuela frente a la dictadura del presidente Nicolás Maduro y la migración de venezolanos hacia el Ecuador. Revista Caribeña de Ciencias Sociales, abril. Recuperado de https://www.eumed.net/rev/caribe/2019/04/ crisis-humanitaria-venezuela.html

6. Chaves, Milciades. (1951). Emigración guajira. Boletín de la Sociedad Geográfica de Colombia, Ix (1), pp. 5-28. Recuperado de https://sogeocol.edu.co/ documentos/009_01_Emigr_guajia.pdf

7. Colombia. Congreso. Ley 191. (23 de junio de 1995). Por medio de la cual se dictan disposiciones sobre Zonas de Frontera. Recuperado de http://www. secretariasenado.gov.co/senado/basedoc/ley_0191_1995.html

8. Colombia. Dirección Nacional de Impuestos y Aduanas Nacionales. Resolución 01922. (23 de febrero de 2007). Por la cual se reglamenta parcialmente el artículo $2 .^{\circ}$ de la Resolución 1890 de 2005. Recuperado de https://www.dian.gov.co/ normatividad/Normatividad/Resoluci\%C3\%B3n\%20001922\%20de\%2023-02-2007. pdf 
9. Defensoría del Pueblo Colombia. (2014). Crisis humanitaria en La Guajira 2014. Acción integral de la Defensoría del Pueblo en el departamento. Bogotá, D.C.: Defensoría del Pueblo.

10. Dover, Robert VH y Puerta Silva, Claudia. (2008). El derecho a la salud: la participación en el régimen subsidiado. Medellín: Universidad de Antioquia.

11. El Heraldo. (2014, septiembre 27). «110 mujeres wayuu están detenidas en Venezuela por contrabando»: Olimpia Palmar. Recuperado de https://www.elheraldo. co/la-guajira/110-mujeres-wayuu-estan-detenidas-en-venezuela-por-contrabandoolimpia-palmar-167861

12. Gandini, Luciana; Prieto, Victoria y Lozano Ascencio, Fernando. (2019). El éxodo venezolano: migración en contextos de crisis y respuestas de los países latinoamericanos. En: Gandini, Luciana; Lozano Ascencio, Fernando y Prieto, Victoria (coords.). Crisis y migración de población venezolana. Entre la desprotección y la seguridad jurídica en Latinoamérica (pp. 9-31). México, D. F.: Universidad Nacional Autónoma de México.

13. González-Plazas, Santiago. (2008). Pasado y presente del contrabando en La Guajira. Aproximaciones al fenómeno de la ilegalidad en la región. Bogotá, D. C.: Universidad del Rosario.

14. Guarnizo, José. (2014, julio 19). ¡La Guajira S. O. S! Semana. Recuperado de http://www.semana.com/nacion/articulo/los-ninos-de-la-guajira-mueren-dehambre/396290-3

15. Guerra, Weildler. (2007). Estudio sobre el desarrollo de la zona de integración fronteriza (ZIF) entre el departamento de La Guajira en Colombia y el estado de Zulia en Venezuela: antecedentes e identificación de temas y proyectos prioritarios. Cartagena de Indias: Observatorio del Caribe Colombiano.

16. Guerrero Barriga, Sandra. (2014, abril 7). Mueren otros dos niños por desnutrición en La Guajira. El Heraldo. Recuperado de http://www.elheraldo.co/laguajira/mueren-otros-dos-ninos-por-desnutricion-en-la-guajira-\%0A148500

17. Gutiérrez de Pineda, Virginia. (1948). Organización social en La Guajira. Estudio etnográfico. Revista del Instituto Etnológico Nacional, 3 (2), 272. Recuperado de http://kt.micrositios.net/action.php?kt_path_info = ktcore.actions.document.view\&f Documentld $=16401$ \&forceopen

18. Howe, Paul. (2018). Famine systems: A new model for understanding the development of famines. World Development, 105, pp. 144-155. DOI: 10.1016/j. worlddev.2017.12.028

19. Liga Contra el Silencio. (2018, noviembre 30). La migración wayuu aumenta la presión en La Guajira. Cuestión Pública. Recuperado de https://cuestionpublica. com/migracion-wayuu-presion-guajira/ 
20. López Ávila, Ana Karen. (2019). El rol del alto comisionado de las Naciones Unidas para los refugiados (ACNUR) en la crisis migratoria de Venezuela 2014-2017. El papel del Estado brasileño. [Tesis inédita de pregrado]. Universidad del Azuay, Cuenca. Recuperado de http://dspace.uazuay.edu.ec/bitstream/datos/8919/1/14567 esp.pdf

21. López Diaz, Jorge Alberto. (2019). Migración Venezolana en Colombia: un desafío para la seguridad. [Tesis inédita de pregrado]. Universidad Militar Nueva Granada, Bogotá, D. C. Recuperado de https://repository.unimilitar.edu. co/bitstream/handle/10654/32380/L\%C3\%B3pezDiazJorgeAlberto2019.pdf. pdf? sequence $=1$ \&isAllowed $=y$

22. Noticias RCN. (2014, enero 27). Restricciones de remesas en Venezuela afecta a colombianos. Noticias RCN. Recuperado de https://noticias.canalrcn.com/ nacional-economia/restricciones-remesas-venezuela-afecta-colombianos

23. Oficina de Naciones Unidas para la Coordinación de Asuntos Humanitarios (OCHA). (2014). Informe Final MIRA: Alta Guajira-Uribia (La Guajira), Colombia. Desabastecimiento de alimentos en comunidades Wayúu de la Alta Guajira (1821 de febrero 2014). Recuperado de https://www.humanitarianresponse.info/sites/ www.humanitarianresponse.info/files/assessments/140307\%20Informe $\%$ 20final $\% 20$ MIRA\%20La\%20Guajira\%20Final.pdf

24. Oficina de Naciones Unidas para la Coordinación de Asuntos Humanitarios (OCHA). (2015). Informe de situación No. 12 - Colombia: Situación humanitaria en

[112] frontera colombo-venezolana. Recuperado de https://www.humanitarianresponse. info/en/operations/colombia/document/informe-de-situaci\%C3\%B3n-no-12colombia-situaci \%C3\%B3n-humanitaria-en-frontera

25. Organización de las Naciones Unidas para la Agricultura y la Alimentación (FAO). (2011). Una introducción a los conceptos básicos de la seguridad alimentaria. Recuperado de http://www.fao.org/docrep/014/al936s/al936s00.pdf

26. Organización de las Naciones Unidas para la Alimentación y la Agricultura (FAO). (2016). Monitoreo de la seguridad alimentaria y nutricional como apoyo a políticas públicas en América Latina y el Caribe. Santiago: FAO.

27. Pérez, Luis Adolfo. (2004). Los Wayuu: tiempos, espacios y circunstancias. Espacio Abierto. Cuaderno Venezolano de Sociología, 13 (4), pp. 607-630. Recuperado de http://www.produccioncientificaluz.org/index.php/espacio/article/view/2115

28. Perrin, Michel. (1992). El camino de los indios muertos. Mitos y símbolos guajiros. Caracas: Monte Ávila.

29. Perrin, Michel. (2003). Guerras Internas. El caso wayuu. Antropológica, (99-100), 143-151. Recuperado de http://www.fundacionlasalle.org.ve/userfiles/Ant 2003 No 99-100 p 143-151.pdf 
30. Picon, François-René. (1983). Pasteurs du nouveau monde. Adoption de l'élevage chez les Indiens guajiros. Paris: Éditions de la Maison des Sciences de I'Homme. DOI: 10.4000/books.editionsmsh.5875

31. Pineda Giraldo, Roberto. (1947). Informe preliminar sobre aspectos sociales y económicos de La Guajira. Expedición 1947. Boletín de Arqueología, 2 (5-6), pp. 529572. Recuperado de https://www.icanh.gov.co/recursos_user//ICANH\%20PORTAL/ PUBLICACIONES/VOL.\%2037\%20(1)/Vol.\%202,\%20n.\%205-6e.pdf

32. Puerta Silva, Claudia et al. (2017). Proyecto: Diseño de un Sistema de Información para el Monitoreo Integral de los Determinantes del Hambre (SINHambre): caso piloto La Guajira indígena. Medellín.

33. Puerta Silva, Claudia y Dover, Robert VH. (2007). Desarrollo de un modelo de interlocución para el sistema de salud basado en el caso de la Guajira colombiana. Revista de Salud Pública, 9 (3), pp. 353-368. DOI: 10.1590/S012400642007000300004

34. Puerta Silva, Claudia. (2013). Stratégies et politiques de reconnaissance et d'identité. Les Indiens wayuu et le projet minier du Cerrejón en Colombie. Bruxelles: P. I. E. Peter Lang. DOI: 10.3726/978-3-0352-6304-6

35. Puerta Silva, Claudia. (2018). Los mapas del hambre: Los wayuu de la Guajira colombiana en la intersección de las geografías de la riqueza y de la exclusión. En: Piazzini Suárez, Carlo Emilio y Montoya Arango, Vladimir (eds.). Cartografías. Nuevos mapas y contramapas. Medellín: Universidad de Antioquia.

36. Raffalli A., Susana y Padrilla, Gonzalo (coords.). (2014). Mapeo de la situación de los medios de vida y la seguridad alimentaria de familias vulnerables en la Alta Guajira. Uribia: Oxfam. Recuperado de https://www.humanitarianresponse.info/ sites/www.humanitarianresponse.info/files/documents/files/diagnostico_seguridad_ alimentaria_alta_guajira_2015_oxfam2.pdf

37. Reina, Mauricio; Mesa, Carlos Antonio y Ramírez Tobón, Tomás. (2018). Elementos para una política pública frente a la crisis de Venezuela. Cuadernos Fedesarrollo (69). Recuperado dehttps://www.repository.fedesarrollo.org.co/bitstream/ handle/11445/3716/CDF_No_69_Noviembre_2018.pdf? sequence $=1$ \&isAllowed $=y$

38. Semana. (2014, julio 19). ¿Estamos perdiendo La Guajira? Recuperado de http://www.semana.com/nacion/articulo/estamos-perdiendo-la-guajira/396289-3

Semana. (2018, octubre 2). En cinco olas, así ha sido la histórica migración de venezolanos. Recuperado de https://www.semana.com/nacion/articulo/crisis-envenezuela-migracion-historica-de-venezolanos-a-colombia/556758

40. Servicio Jesuita a Refugiados (SJR). (2015). Venezuela: una aproximación a la situación actual desde la opinión pública. Revista SIC. Recuperado de http:// revistasic.gumilla.org/2015/venezuela-una-aproximacion-a-la-situacion-actual-desdela-opinion-publica/ 
41. Sutherland, Manuel. (2019). Hiperinflación, crisis, trabajo asalariado, poder adquisitivo. Crónica de una ruina anunciada. Cuadernos del Cendes, 36 (100), pp. 35-55.

43. Viloria de la Hoz, Joaquín. (2014). Negocios en la frontera: agricultura, comercio y actividad extractiva en La Guajira colombiana, 1870-1930. Caribbean Studies, 42 (1), pp. 183-224. DOI: 10.1353/crb.2014.0018

44. Zúñiga Hernández, María Alejandra. (2015). El reto de la seguridad alimentaria en el municipio de Uribia-La Guajira. Una mirada desde la gobernabilidad. [Tesis inédita de pregrado]. Recuperado de https://repository.javeriana.edu.co/handle/10554/19034 